\title{
Dengue virus surveillance: circulation of DENV-1, DENV-2, DENV- 3 and DENV-4 in mosquitoes around Taman Connaught, Cheras
}

\author{
Wen Li Heng ${ }^{a}$, Kin Yen Chia, ${ }^{\mathrm{a}, \mathrm{b}}$, Ismail Nashwaan ${ }^{\mathrm{a}}$, Hui Yee Chee ${ }^{\mathrm{c}}$, Crystale Siew Ying Limª \\ ${ }^{a}$ Department of Biotechnology, Faculty of Applied Sciences, UCSI University Kuala Lumpur Campus, No. 1, Jalan Menara Gading, UCSI \\ Heights (Taman Connaught), Cheras, 56000 Kuala Lumpur, Malaysia \\ ${ }^{b}$ Department of Applied Biomedical Science and Biotechnology, Faculty of Health Sciences, International Medical University, Bukit Jalil, 57000 \\ Kuala Lumpur, Malaysia \\ 'Department of Medical Microbiology and Parasitology, Faculty of Medicine \& Health Sciences, Universiti Putra Malaysia, 43400 Serdang, \\ Selangor, Malaysia
}

Received 10th June 2019 / Accepted 8th October 2019

\begin{abstract}
Dengue, a mosquito-borne viral infection that causes dengue fever, hemorrhagic fever and dengue shock syndrome, is on a drastic rise in recent years. In Malaysia, the distribution of dengue viruses is determined based on localized studies, where these data are essential to predict the epidemiology of dengue infections. Therefore, a study was conducted in Taman Connaught, Cheras, determining dengue virus serotypes in field caught Aedes mosquitoes. Out of 1,147 mosquitoes captured from September to December 2017, 156 Aedes spp. mosquitoes were identified based on their physical features using a digital microscope. Total RNA was then extracted from the head and thorax of the mosquitoes and subjected to reverse transcriptase-polymerase chain reaction (RT-PCR) with DENV-1, DENV-2, DENV-3 and DENV-4 primer sets, respectively. The results show that at least one DENV serotype was detected in 91\% ( $\mathrm{n}=142)$ out of 156 Aedes spp. mosquitoes. The predominant serotype detected was DENV-2 [47.44\% $(\mathrm{n}=74)]$, followed by DENV-4 [8.97\% $(\mathrm{n}=14)]$, DENV-3 [7.05\% $(\mathrm{n}=11)]$ and DENV-1 [3.85\% $(\mathrm{n}=6)]$, respectively. Co-circulation of DENV-2 and -4 was the predominant co-infection detected in Aedes spp. mosquitoes. We also present the first report of DENV-1, -2 and -4 co-infection in field-caught Aedes spp. Localized surveillance mechanisms based on serotype circulation in the mosquito vector can be an effective monitoring system for the government and public health sector in order to reduce the incidence of dengue hotspots.
\end{abstract}

Keywords: Aedes spp., dengue virus, RT-PCR, serotypes, surveillance

\section{INTRODUCTION}

The Aedes mosquito is the vector for the dengue virus, which is the etiological agent for dengue fever, hemorrhagic fever and dengue shock syndrome. Dengue is now the most epidemiologically-important viral mosquitoborne disease in the world, with a steady increase in the number of dengue cases globally (World

*Author for correspondence: Crystale Lim Siew Ying, Department of Biotechnology, Faculty of Applied Sciences, UCSI University Kuala Lumpur Campus, No. 1, Jalan Menara Gading, UCSI Heights (Taman Connaught), Cheras, 56000 Kuala Lumpur, Malaysia.Email - crystalelim@ucsiuniversity. edu.my
Health Organization, 2019; Armstrong et al., 2003).

Four dengue virus serotypes, DENV-1, DENV-2, DENV-3 and DENV-4, found at different frequencies in different geographic regions, are associated with the epidemic potential of DENV (Quintero-Gil et al., 2014). DENV serotypes use the same putative receptors in the 
mosquito midgut, where the susceptibility of DENV infection in mosquitoes can alter the accuracy of predictions on DENV transmission (Carrington et al., 2014). Some viral variants may have a strong fitness advantage, such as better ability to produce and or utilize genetically different polymerases or capsid proteins (Pepin et al., 2008). This intra-host competition within the mosquito vector can occur between different serotypes of the same virus (Quintero-Gil et al., 2014).

Virus surveillance is crucial in detecting and predicting infection outbreaks, where it is an important infection-control tool to understand spatial or temporal infection dynamics and evaluate control interventions (Pérez-Castro et al., 2016). Virus surveillance can also serve as an important mechanism in the identification of viruses with higher virulence in studies of natural viral evolution (Cologna et al., 2005).

In Malaysia, the Dengue Situation Update for July 2019 reported a half-year incidence of 62,421 cases as of 29th June 2019, which is nearly double the incidence reported for the same duration in 2018 (World Health Organization, 2019). The extraordinary rise of dengue cases from year to year has been reported in previous studies where records show a $160 \%$ increase in dengue cases reported within the Kuala Lumpur metropolitan area from 2015 to 2016. Of a total of 8664 dengue cases reported in 2016, the Cheras district contributed 20\% of these dengue cases (Mahmud et al., 2018) in comparison to other urban districts within Kuala Lumpur. Cheras was previously reported with the highest number of dengue cases in Kuala Lumpur, with the prevalence of all four DENV serotypes (Chew et al., 2012).

Hence, data on vector carriage may be useful in developing effective intervention strategies in dengue hotspot areas. Thusly, we report the surveillance of dengue-infected mosquito populations around Taman Connaught in Cheras to identify dengue virus serotypes at risk for transmission from vectors to humans.

\section{MATERIALS AND METHODS}

\section{Specimen collection and identification}

Convenience sampling of 1,147 field-caught mosquitoes was carried out from September 2017 to December 2017 (4 months) within a one $\mathrm{km}$ radius sampling area in Taman Connaught, Cheras which covered commercial, residential and recreational facilities. Five commercial ultraviolet light-emanating mosquito traps were placed at sheltered strategic locations around the sampling area, along building corridors with a high frequency of daily human activity. Mosquitoes were continuously collected in the mornings between 8 -10am throughout the 4 months, stored in sterile containers kept on ice, then transported to the laboratory for species identification on the same day. Mosquitoes were categorized into Aedes and non-Aedes mosquitoes using a digital microscope (Dino-Lite Edge, AnMo Electronics Corporation, Taiwan) at $80 \mathrm{X}$ magnification, where the physical morphology of mosquitoes was determined based on the guidelines of Walter Reed Biosystematics Unit, by accessing information available at http://www.wrbu.org/ $\mathrm{mqID} / \mathrm{mq}$ gnra/aedes.htm. Once identified, mosquitoes were stored at $-80^{\circ} \mathrm{C}$ prior to RNA extraction.

\section{$\boldsymbol{R N A}$ extraction and reverse transcription}

Viral titers of dengue are high in the head, thorax (where the salivary glands are located) and midgut of the mosquito (Tsai et al., 2017), which provide reliable dengue positivity screening via molecular methods (Pankhong et al., 2002; Thavara et al., 2006). Thus, in the present study, the head and thorax of individual Aedes mosquitoes were dissected using a forceps and scalpel under a digital microscope (Dino-Lite Edge, AnMo Electronics Corporation, Taiwan) and used for subsequent reverse-transcription Polymerase Chain Reaction (RT-PCR). RNA extraction was done for the head and thorax of individual mosquitoes using TRIzol reagent (Thermo Fisher Scientific, USA) according to the manufacturer's protocol. Reverse transcription was then carried out in $20 \mu \mathrm{L}$ reactions. Briefly, $0.5 \mu \mathrm{g}$ of total extracted RNA and $1 \mu \mathrm{L}$ of $0.2 \mu \mathrm{g}$ of $100 \mathrm{pmol}$ random hexamers (Thermo Fisher Scientific, USA) were added to PCR tube and topped up 
with $0.1 \%$ DEPC water. Subsequently, the mixture was added with $4 \mu \mathrm{L}$ of $5 \mathrm{X}$ reaction buffer, $0.5 \mu \mathrm{L}$ of $20 \mathrm{U} / \mu \mathrm{L}$ RNase inhibitor, $2 \mu \mathrm{L}$ of $10 \mathrm{mM}$ dNTP, and $1 \mu \mathrm{L}$ of $200 \mathrm{U}$ MMLVReverse Transcriptase (all from Thermo Fisher Scientific, USA). The resulting reversetranscribed cDNA was used for subsequent PCR confirmation of Aedes spp. and PCR screening of DENV serotypes 1 to 4 .

\section{PCR confirmation of Aedes spp.}

PCR primers to amplify the consensus region of the small unit ribosomal RNA (18S rRNA) gene of Aedes spp (as in Table 1) were designed based on the GenBank IDs AY988440.1 (A. aegypti) and HQ010437 (A. albopictus), respectively. PCR was carried out in $25 \mu \mathrm{L}$ reactions with $2.5 \mu \mathrm{L}$ of $10 \mathrm{X}$ Standard Taq Reaction Buffer, $0.5 \mu \mathrm{L}$ of $10 \mathrm{mM}$ dNTP, $0.125 \mu \mathrm{L}$ of $5000 \mathrm{U} / \mathrm{mL}$ Taq DNA polymerase (all from New England Biolabs, USA), $0.5 \mu \mathrm{L}$ of each $10 \mu \mathrm{M}$ forward and reverse primers (IDT, Singapore), and $1.0 \mu \mathrm{L}$ of reversetranscribed cDNA, and then topped up with nuclease-free water. The PCR reactions were subjected to the following cycling conditions in a thermal cycler (Nexus Gradient, Eppendorf, Germany): initial denaturation at $95^{\circ} \mathrm{C}$ for $30 \mathrm{~s}$; then 35 cycles of denaturation at $95^{\circ} \mathrm{C}$ for $15 \mathrm{~s}$, annealing at $53.3^{\circ} \mathrm{C}$ for $30 \mathrm{~s}$, and extension at $68^{\circ} \mathrm{C}$ for $30 \mathrm{~s}$; followed by a final extension at $68^{\circ} \mathrm{C}$ for 5 min. PCR products were analyzed using 1\% agarose gel electrophoresed in standard Tris Borate EDTA buffer at $80 \mathrm{~V}$ for $30 \mathrm{~min}$, and visualized under ultraviolet light (Quantum ST4 Transilluminator + Quantum Capt Softwares, Viber Lourmat, France). Representative PCR products were gel-purified (GF-1 Gel DNA Recovery Kit, Vivantis Technologies Sdn. Bhd, Malaysia) for DNA sequencing (MyTACG, Taiwan) with the respective forward and reverse primers for PCR amplicon confirmation.

\section{PCR screening of DENV-1 to DENV-4}

PCR primers previously designed by Tanaka (1993) were used to detect DENV-1, DENV-2, DENV-3 and DENV-4 in Aedes spp.-positive samples. PCR was carried out using the cDNA of individual mosquitoes as before, with the exception of the specific DENV primers and the respective annealing temperature (Table 1). Every PCR run for a specific DENV included the respective DENV-specific positive control (a kind gift from Dr. Chee Hui Yee of Universiti Putra Malaysia), and a no-template control. DENV positive controls and representative were confirmed for primer specificity via DNA sequencing with the respective DENV primers.

Table 1. All PCR primers used in this study.

\begin{tabular}{|c|c|c|c|}
\hline Primers & Sequences (5'-3') & $\begin{array}{c}\text { Optimized } \\
\text { annealing } \\
\text { temperature }\left({ }^{\circ} \mathrm{C}\right)\end{array}$ & $\begin{array}{l}\text { PCR product } \\
\text { size (bp) }\end{array}$ \\
\hline $\begin{array}{l}\text { Aedes spp. } \\
\text { 18S rRNA }\end{array}$ & $\begin{array}{l}\text { F:5'-CATCGTGGT'TGACT'TCTGCT-3' } \\
\text { R: 5'-AAAGGGCAGGGACGTAATC-3' }\end{array}$ & 53.3 & 262 \\
\hline DENV-1 ${ }^{[16]}$ & $\begin{array}{l}\text { F: 5'-GGACTGCGTATGGAGTTTTG-3' } \\
\text { R: 5'-ATGGGTTGTGGCCTAATCAT-3' }\end{array}$ & 49 & 490 \\
\hline DENV-2 ${ }^{[16]}$ & $\begin{array}{l}\text { F: 5'-GTTCCTCTGCAAACACTCCA-3' } \\
\text { R: 5'-GTGTTATTTTGAT'T'TCCT'TG-3' }\end{array}$ & 45 & 230 \\
\hline DENV-3 ${ }^{[16]}$ & $\begin{array}{l}\text { F: 5'-GTGCTTACACAGCCCTATT'T-3' } \\
\text { R: 5'-TCCATTCTCCCAAGCGCCTG-3' }\end{array}$ & 51.5 & 320 \\
\hline DENV-4 ${ }^{[16]}$ & $\begin{array}{l}\text { F: 5'-CCAT'TATGGCTGTGTTGT'T'T-3' } \\
\text { R: 5'-CTTCATCCTGCTTCACTTCT-3' }\end{array}$ & 46 & 398 \\
\hline
\end{tabular}

\section{RESULTS}

Out of a total of 1,147 mosquitoes caught in this study, 156 were morphologically identified as
Aedes spp., of which $74.7 \%$ were $A$. aegypti while $18.6 \%$ were $A$. albopictus.

The average concentration of the extracted RNA from the 156 Aedes spp. individuals was 90 $\mathrm{ng} / \mu \mathrm{L}$. Reverse transcription-PCR amplification 
with 18S rRNA primers specific for Aedes spp. confirmed the morphological identification of all individuals as Aedes spp. (Figure 1).

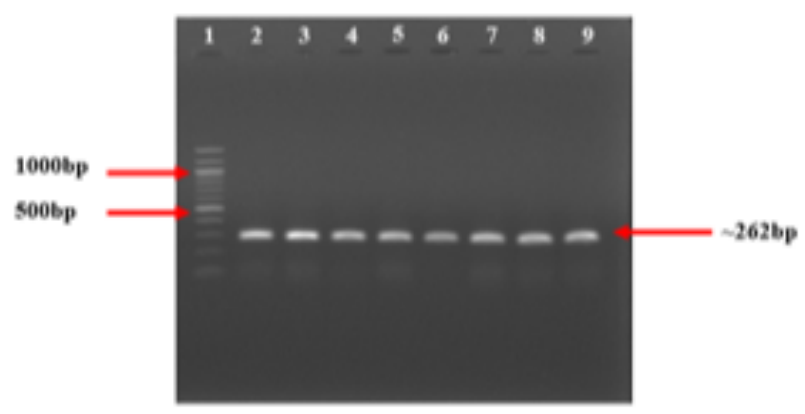

Figure 1. Representative agarose gel electrophoresis image of Aedes spp. $18 \mathrm{~S}$ rRNA PCR products. Lanes 2 to 9: PCR products of samples positive for the Aedes spp. $18 \mathrm{~S}$ rRNA ( 262bp), Lane 1: 100bp DNA ladder marker (New England Biolabs, USA).

Subsequent PCR with DENV-1 to DENV-4 primers specific to the respective dengue virus serotypes (Figures $2 \mathrm{~A}$ and $2 \mathrm{~B}$ ) revealed that out of 156 Aedes spp., DENV-2 was the most prevalent single serotype detected [47.4\%, $\mathrm{n}=74]$, followed by DENV-4 [9\% $(n=14)]$, DENV-3 $[7.1 \% \quad(\mathrm{n}=11)]$ and DENV-1 [3.8\% $(\mathrm{n}=6)]$, respectively (Table 2$)$. Fourteen mosquitoes $(9 \%)$ did not carry any of the four dengue virus serotypes.

Our results also revealed multiple dengue virus serotypes in 37 mosquitoes (23.7\%), where DENV-2 + DENV-4 was the predominant coinfection detected (Table 3), occurring in 12 individuals. Co-detection of up to three dengue virus serotypes (DENVs-1, -2 and -4) was observed in four mosquitoes.

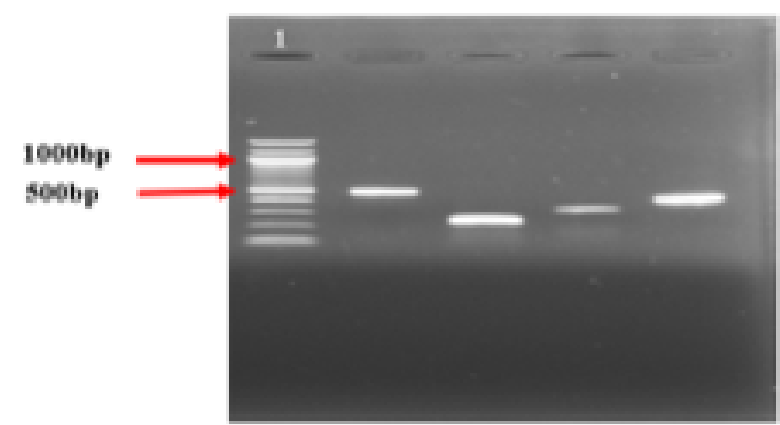

Figure 2A. Agarose gel electrophoresis picture for DNA of positive controls, from P1 to P4, tested with four dengue primer pairs through PCR reaction. Lane 2 to 5: PCR products of positive controls of DENV-1 to DENV-4, for P1 ( 490bp), P2 ( 230bp), P3 ( 320bp), and P4 ( 398bp), Lane 1: 100bp DNA ladder marker (NEB, USA).

Table 2. Aedes spp. and DENV serotypes detected in this study. Aedes aegypti is the predominant Aedes spp. $(74.4 \%)$, while DENV-2 is the predominant serotype $(47.4 \%)$ in this study.

\begin{tabular}{|c|c|c|c|c|c|c|c|}
\hline \multirow[b]{2}{*}{ Aedes species } & \multicolumn{6}{|c|}{ DENV serotypes detected in individual mosquitoes [\%] } & \multirow[b]{2}{*}{ Total } \\
\hline & $\begin{array}{c}\text { DENV- } \\
1\end{array}$ & $\begin{array}{c}\text { DENV } \\
-2\end{array}$ & $\begin{array}{c}\text { DENV } \\
-3\end{array}$ & $\begin{array}{c}\text { DENV } \\
-4\end{array}$ & $\begin{array}{c}\text { Co- } \\
\text { detected } \\
\mathrm{DENV}^{\wedge}\end{array}$ & No DENV & \\
\hline A. aegypti & $\begin{array}{c}5 \\
{[3.2]}\end{array}$ & 48 [30.8] & $\begin{array}{c}10 \\
{[6.4]}\end{array}$ & $\begin{array}{c}14 \\
{[9.0]}\end{array}$ & $\begin{array}{c}27 \\
{[17.3]}\end{array}$ & $\begin{array}{c}12 \\
{[7.7]}\end{array}$ & $\begin{array}{c}116 \\
{[74.4] .}\end{array}$ \\
\hline A. albopictus & 0 & $\begin{array}{c}19 \\
{[12.2]}\end{array}$ & $\begin{array}{c}1 \\
{[0.6]}\end{array}$ & 0 & $\begin{array}{c}7 \\
{[4.5]}\end{array}$ & $\begin{array}{c}2 \\
{[1.3]}\end{array}$ & $\begin{array}{c}29 \\
{[18.6]}\end{array}$ \\
\hline Other* & $\begin{array}{c}1 \\
{[0.6]}\end{array}$ & $\begin{array}{c}7 \\
{[4.5]}\end{array}$ & 0 & 0 & $\begin{array}{c}3 \\
{[1.9]}\end{array}$ & 0 & $\begin{array}{c}11 \\
{[7.1]}\end{array}$ \\
\hline Total & $\begin{array}{c}6 \\
{[3.8]}\end{array}$ & $\begin{array}{c}74 \\
{[47.4]}\end{array}$ & $\begin{array}{c}11 \\
{[7.1]}\end{array}$ & $\begin{array}{c}14 \\
{[9.0]}\end{array}$ & $\begin{array}{c}37 \\
{[23.7]}\end{array}$ & $\begin{array}{c}14 \\
{[9.0]}\end{array}$ & 156 \\
\hline
\end{tabular}

*Other Aedes spp.: A. togoi and A. psendonivens

${ }^{\wedge}$ Refer to Table 3 
Table 3. DENV serotypes co-detected in this study. DENV-2 + DENV-4 are the predominant codetected serotypes $(32.4 \%)$ in this study.

\begin{tabular}{ccccc}
\hline \multirow{2}{*}{ DENV serotype } & \multicolumn{4}{c}{ Mixed dengue serotypes detected in individual mosquitoes [\%] } \\
\cline { 2 - 5 } & DENV-1 & DENV-2 & DENV-3 & DENV-4 \\
\hline DENV-1 & $/$ & $2[5.4]$ & $1[2.7]$ & - \\
\hline DENV-2 & $6[16.2]$ & $/$ & - & $1[2.7]$ \\
\hline DENV-3 & - & $2[5.4]$ & $/$ & $4[10.8]$ \\
\hline DENV-4 & $1[2.7]$ & $12[32.4]$ & $4[10.8]$ & $/$ \\
\hline DENV-1 + DENV-2 & - & - & - & $4[10.8]$ \\
\hline
\end{tabular}

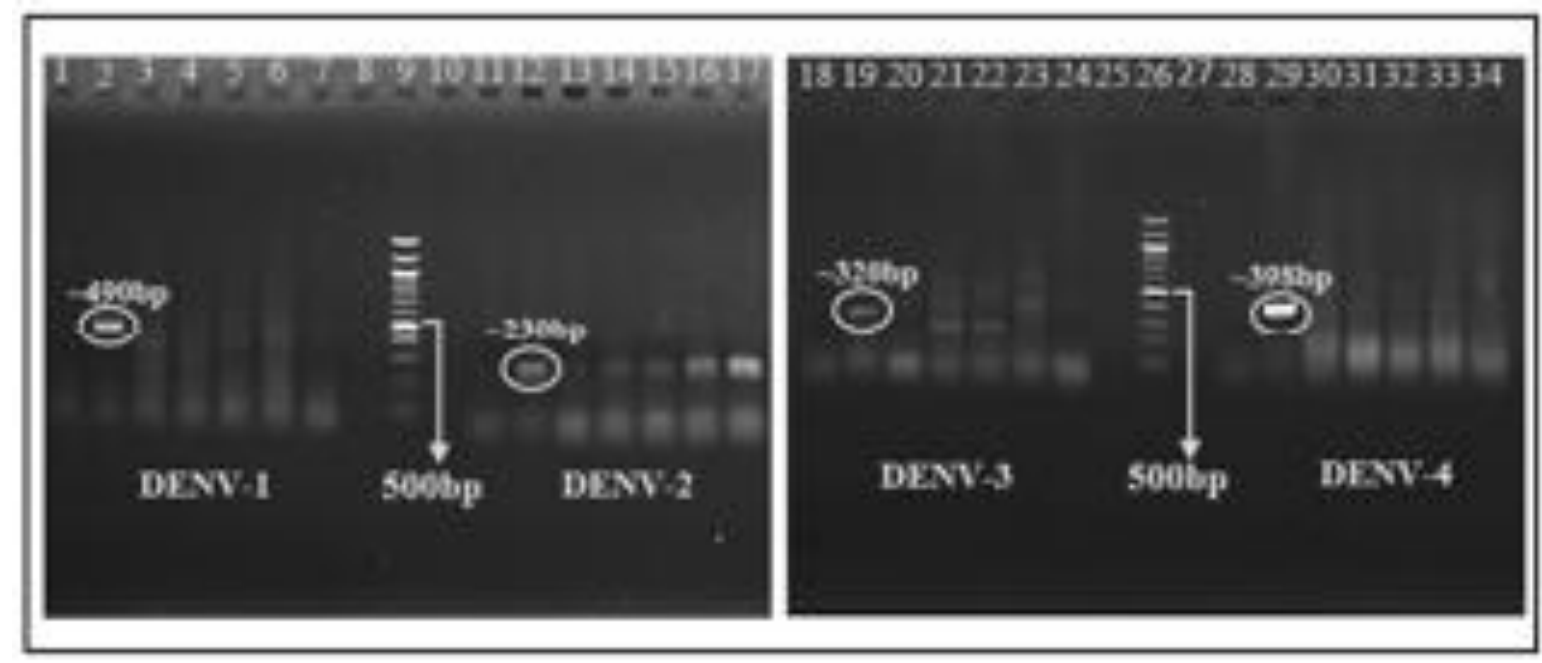

Figure 2B. Agarose gel electrophoresis image for DENV-1 to DENV-4 primer sets testing on representative mosquito. Lane 3 to 7, Lane 13 to 17, Lane 20 to 24, and Lane 30-34: PCR products of Mos 7, 9, 10, 11, and 12 tested by DENV-1 to DENV-4 primer sets, respectively. Lane 1, Lane 11, Lane 18 and Lane 28: negative controls. Lane 2, Lane 12, Lane 19, and Lane 29: positive controls, P1 to P4. Lane 9 and 26: 100bp DNA ladder marker (NEB, USA).

\section{DISCUSSION}

Of all 156 Aedes spp. mosquitoes caught, 142 tested positive for single or multiple dengue virus serotypes. The number of $A$. aegypti captured as compared to $A$. albopictus in this study is consistent with the general consensus that $A$. aegypti is gradually overtaking the native species of $A$. albopictus within the region (Rudnick, 1965). While $A$. aegypti is considered the primary vector for dengue, both species are significant vectors for arboviruses and are capable of carrying multiple arbovirus infections such as Chikungunya and Zika (Cogan, 2019).

A previous study in a hospital setting in Cheras found that although co-circulation of all four DENV serotypes were present, DENV-4 was the predominant serotype within 46 dengue positive cases with $97 \%$ being single or dual infection with DENV-4 (Chew et al., 2012). The present study, which is the first report of dengue virus serotypes in Aedes spp. in Cheras, found that DENV-2 (47.4\%) was the predominant serotype in the vector instead, followed by DENV-4 (9\%).

From general clinical data, there is observed seasonal drift in dominant dengue serotypes over the years with DENV-3 dominating in 2014 and DENV-4 gaining prominence in recent years, whilst still constituting less than $20 \%$ of all serotypes between 2000-2012 (Chew et al., 2012; Mohd-Zaki et al., 2014; Cogan, 2019). However, there has yet to be a clear correlation between the dominant DENV serotype found in infected humans and the dominant DENV serotype within mosquitoes, as infection dynamics depend 
on the number of infected mosquitoes, proximity to humans, weather patterns, virological factors and other elements (Bar-Zeev et al., 1977; Cogan, 2019)

Co-infections are associated with more severe clinical manifestations of the disease, most notably with increased likelihood of developing Dengue Hemorrhagic Fever (DHF) (Gubler, 1998). The circulation of multiple DENV serotypes in mosquito vectors may increase both the occurrence of co-infection within humans and also the risk of subsequent secondary infection with a different dengue serotypes, leading to poorer prognoses (Dhanoa et al., 2016). DENV-2 + DENV-3 co-infections in individual mosquitoes (10 mosquitoes with DENV-2 + DENV-3 of 15 total co-infections) were previously reported in Selangor (Lau et al., 2015).

In a 2016 study conducted in Johor Bahru (Malaysia) using 262 Dengue positive patient sera samples, $40(15 \%)$ of samples were identified as co-infections (with DENV-1/DENV-2 contributing $85 \%$ of co-infections) (Dhanoa et al., 2016). The prevalence of several serotypes within a community or region collectively increases the impact of seasonal dengue outbreaks and coinfections and monitoring of dengue virus within the mosquito population and subsequent control measures may prove to be a vital precautionary step and serve as an early warning system to thwart the increasing burden of dengue outbreaks.

The present study found serotype coinfections in 37 individual Aedes spp. mosquitoes, with DENV-2 + DENV-4 as the prevalent coinfection. Interestingly, our study reports four individual Aedes spp. mosquitoes co-infected with three DENV serotypes (DENV-1, -2 and -4) which, to our knowledge, is the first such report of tri-DENV co-infections in field-caught Aedes spp.

\section{CONCLUSION}

In conclusion, this study suggests that DENV-2 is the predominant DENV serotype in the Taman Connaught area, with a large number of mosquitoes carrying co-infections of dengue serotypes. Data on localized DENV serotype circulation in mosquito vectors has the potential provide an effective monitoring mechanism to the government, public health officials and the general public in order to develop better containment and dengue eradication strategies targeting the dengue mosquito vector.

\section{ACKNOWLEDGEMENTS}

This project was supported in part by UCSI University's CERVIE Pioneer Scientist Incentive Fund (PSIF) project No. Proj-In-FAS-023.

\section{REFERENCES}

Armstrong, P. M. \& Rico-Hesse, R. 2003. Efficiency of dengue serotype 2 virus strains to infect and disseminate in Aedes aegypti. American Journal of Tropical Medicine and Hygiene 68(5): 539-544.

Bar-Zeev, M., Maibach, H. I., \& Khan, A. A. 1977. Studies on the attraction of Aedes aegypti (Diptera: Culicidae) to man. Journal of Medical Entomology 14(1): 113-120.

Carrington, L. B. \& Simmons, C. P. 2014. Human to mosquito transmission of dengue viruses. Frontiers in Immunology 5 : 290.

Cogan, J. 2019. Dengue and severe dengue, World Health Organization.

Cologna, R., Armstrong, P. M., \& Rico-Hesse, R. 2005. Selection for virulent dengue viruses occurs in humans and mosquitoes. Journal of Virology 79(2): 853-859.

Dhanoa, A. et al. 2016. Impact of dengue virus (DENV) coinfection on clinical manifestations, disease severity and laboratory parameters. BMC Infectious Diseases 16(1): 406.

Gubler, D. J. 1998. Dengue and dengue hemorrhagic fever. Clinical Microbiology Reviews 11(3): 480-496.

H Chew, M. et al. 2012 All serotypes of dengue viruses circulating in Kuala Lumpur, Malaysia. Current Research Journal of Biological Sciences 4: 229-234.

Lau, S.-M. et al. 2015. Surveillance of adult Aedes mosquitoes in Selangor, Malaysia. Tropical Medicine \& International Health 20(10): 1271-1280.

Mahmud, M. A. F. et al. 2018. Study on key Aedes spp breeding containers in dengue outbreak localities in Cheras district, Kuala Lumpur. International Journal of Mosquito Research 5(2): 23-30.

Mohd-Zaki, A. H. et al. 2014. Epidemiology of dengue disease in Malaysia (2000-2012): A systematic literature review. PLoS Neglected Tropical Diseases 8(11): 3159.

Pankhong, P. et al. 2002. Molecular serotyping of dengue viruses in field-caught Aedes mosquitos by in-house RNA extraction/RT-PCR reagent kits. The Southeast Asian Journal of Tropical Medicine and Public Health 33(3): 139-144.

Pepin, K. M., Lambeth, K., \& Hanley, K. A. 2008. Asymmetric competitive suppression between strains of dengue virus. BMC Microbiology 8(1): 28.

Pérez-Castro, R. et al. 2016. Detection of all four dengue serotypes in Aedes aegypti female mosquitoes collected in a rural area in Colombia. Memórias do Instituto Oswaldo Cruz 111(4): 233 240. 
Quintero-Gil, D. C. et al. 2014. Differential replication of dengue virus serotypes 2 and 3 in coinfections of C6/36 cells and Aedes aegypti mosquitoes. The Journal of Infection in Developing Countries 8(07): 876-884.

Rudnick, A. 1965. Studies of the ecology of dengue in Malaysia: A preliminary report. Journal of Medical Entomology 2(2): 203 208.

Tanaka, M. 1993. Rapid identification of flavivirus using the polymerase chain reaction. Journal of Virological Methods 41(3): 311-322.

Thavara, U. et al. 2006. Double infection of heteroserotypes of dengue viruses in field populations of Aedes aegypti and Aedes albopictus (Diptera: Culicidae) and serological features of dengue viruses found in patients in southern Thailand. The Southeast Asian Journal of Tropical Medicine and Public Health 37(3): 468-476.

Tsai, C. H. et al. 2017. The impact of temperature and Wolbacbia infection on vector competence of potential dengue vectors Aedes aegypti and Aedes albopictus in the transmission of dengue virus serotype 1 in southern Taiwan. Parasites \& Vectors 10(1): 551.

World Health Organization. 2019. Update on the Dengue situation in the Western Pacific Region. 